\title{
Interval Oscillation Criteria for a Class of Fractional Differential Equations with Damping Term
}

\author{
Chunxia Qi and Junmo Cheng \\ School of Business, Shandong University of Technology, Zibo, Shandong 255049, China \\ Correspondence should be addressed to Chunxia Qi; qichunxiasdut@126.com
}

Received 20 January 2013; Accepted 10 March 2013

Academic Editor: Sotiris Ntouyas

Copyright (C) 2013 C. Qi and J. Cheng. This is an open access article distributed under the Creative Commons Attribution License, which permits unrestricted use, distribution, and reproduction in any medium, provided the original work is properly cited.

Some new interval oscillation criteria are established based on the certain Riccati transformation and inequality technique for a class of fractional differential equations with damping term. For illustrating the validity of the established results, we also present some applications for them.

\section{Introduction}

Fractional differential equations are generalizations of classical differential equations of integer order and can find their applications in many fields of science and engineering. In the last few decades, research on various aspects of fractional differential equations, for example, the existence, uniqueness, and stability of solutions of fractional differential equations, the numerical methods for fractional differential equations, and so on, has been paid much attention by many authors (e.g., we refer the reader to see [1-8] and the references therein). In these investigations, we notice that very little attention is paid to oscillation of fractional differential equations. Recent results in this direction only include Chen's work [9], in which some new oscillation criteria are established for the following fractional differential equation:

$$
\begin{aligned}
{\left[r(t)\left(D_{-}^{\alpha} y(t)\right)^{\eta}\right]^{\prime}-q(t) f\left(\int_{t}^{\infty}(v-t)^{-\alpha} y(v) d v\right) } & =0 \\
t & >0
\end{aligned}
$$

where $r, q$ are positive-valued functions and $\eta$ is the quotient of two odd positive numbers.

In this paper, we are concerned with oscillation of solutions of fractional differential equations of the following form:

$$
\left(a(t)\left[r(t) D_{-}^{\alpha} x(t)\right]^{\prime}\right)^{\prime}+p(t)\left[r(t) D_{-}^{\alpha} x(t)\right]^{\prime}
$$

$$
-q(t) \int_{t}^{\infty}(\xi-t)^{-\alpha} x(\xi) d \xi=0, \quad t \in\left[t_{0}, \infty\right)
$$

where $a \in C^{1}\left(\left[t_{0}, \infty\right), \mathbb{R}_{+}\right), r \in C^{2}\left(\left[t_{0}, \infty\right), \mathbb{R}_{+}\right), p, q \in$ $C\left(\left[t_{0}, \infty\right), \mathbb{R}_{+}\right), \alpha \in(0,1), D^{\alpha} x(t)$ denotes the Liouville right-sided fractional derivative of order $\alpha$ of $x$, and $D^{\alpha} x(t)=$ $-1 /(\Gamma(1-\alpha))(d / d t) \int_{t}^{\infty}(\xi-t)^{-\alpha} x(\xi) d \xi$.

A solution of (2) is said to be oscillatory if it is neither eventually positive nor eventually negative; otherwise it is nonoscillatory. Equation (2) is said to be oscillatory in case all its solutions are oscillatory.

The organization of the rest of this paper is as follows. In Section 2, we establish some new interval oscillation criteria for (2) by a generalized Riccati transformation and inequality technique and present some applications for our results in Section 3. Throughout this paper, $\mathbb{R}$ denotes the set of real numbers, and $\mathbb{R}_{+}=(0, \infty)$. For more details about the theory of fractional differential equations, we refer the reader to [1012].

\section{Main Results}

For the sake of convenience, in the rest of this paper, we set $X(t)=\int_{t}^{\infty}(\xi-t)^{-\alpha} x(\xi) d \xi, A(t)=\int_{t_{0}}^{t}(p(s) / a(s)) d s, \delta_{1}(t, a)$ $=\int_{a}^{t}\left(1 / e^{A(s)} a(s)\right) d s$, and $\delta_{2}(t, a)=\int_{a}^{t}\left(\delta_{1}(s, a) / r(s)\right) d s$. 
Lemma 1. Assume $x$ is a solution of (2). Then $X^{\prime}(t)=-\Gamma(1-$ $\alpha) D^{\alpha} x(t)$

Lemma 2. Assume $x$ is an eventually positive solution of (2), and

$$
\begin{gathered}
\int_{t_{0}}^{\infty} \frac{1}{e^{A(s)} a(s)} d s=\infty, \\
\int_{t_{0}}^{\infty} \frac{1}{r(s)} d s=\infty, \\
\int_{t_{0}}^{\infty} \frac{1}{r(\xi)} \int_{\xi}^{\infty} \frac{1}{e^{A(\tau)} a(\tau)} \int_{\tau}^{\infty} e^{A(s)} q(s) d s d \tau d \xi=\infty .
\end{gathered}
$$

Then there exists a sufficiently large $T$ such that

$$
\left[r(t) D_{-}^{\alpha} x(t)\right]^{\prime}<0 \text { on }[T, \infty)
$$

and either $D_{-}^{\alpha} x(t)<0$ on $[T, \infty)$ or $\lim _{t \rightarrow \infty} X(t)=0$.

Proof. Since $x$ is an eventually positive solution of (2), there exists $t_{1}$ such that $x(t)>0$ on $\left[t_{1}, \infty\right)$. So $X(t)>0$ on $\left[t_{1}, \infty\right)$, and we have

$$
\begin{gathered}
\left(e^{A(t)} a(t)\left[r(t) D_{-}^{\alpha} x(t)\right]^{\prime}\right)^{\prime} \\
=e^{A(t)}\left(a(t)\left[r(t) D_{-}^{\alpha} x(t)\right]^{\prime}\right)^{\prime} \\
+e^{A(t)} p(t)\left[r(t) D_{-}^{\alpha} x(t)\right]^{\prime} \\
=e^{A(t)}\left\{\left(a(t)\left[r(t) D_{-}^{\alpha} x(t)\right]^{\prime}\right)^{\prime}\right. \\
\left.+p(t)\left[r(t) D_{-}^{\alpha} x(t)\right]^{\prime}\right\} \\
=e^{A(t)} q(t) X(t)>0 .
\end{gathered}
$$

Then $e^{A(t)} a(t)\left[r(t) D^{\alpha} x(t)\right]^{\prime}$ is strictly increasing on $\left[t_{1}, \infty\right)$, and thus $\left[r(t) D^{\alpha} x(t)\right]^{\prime}$ is eventually of one sign. We claim $\left[r(t) D^{\alpha} x(t)\right]^{\prime}<0$ on $\left[t_{2}, \infty\right)$, where $t_{2}>t_{1}$ is sufficiently large. Otherwise, assume there exists a sufficiently large $t_{3}>$ $t_{2}$ such that $\left[r(t) D_{-}^{\alpha} x(t)\right]^{\prime}>0$ on $\left[t_{3}, \infty\right)$. Then for $t \in\left[t_{3}, \infty\right)$, we have

$$
\begin{aligned}
r(t) D_{-}^{\alpha} x(t)-r\left(t_{3}\right) D_{-}^{\alpha} x\left(t_{3}\right) & \\
& =\int_{t_{3}}^{t} \frac{e^{A(s)} a(s)\left[r(s) D_{-}^{\alpha} x(s)\right]^{\prime}}{e^{A(s)} a(s)} d s \\
& \geq e^{A\left(t_{3}\right)} a\left(t_{3}\right)\left[r\left(t_{3}\right) D_{-}^{\alpha} x\left(t_{3}\right)\right]^{\prime} \int_{t_{3}}^{t} \frac{1}{e^{A(s)} a(s)} d s .
\end{aligned}
$$

By (3), we have

$$
\lim _{t \rightarrow \infty} r(t) D_{-}^{\alpha} x(t)=\infty
$$

which implies for some sufficiently large $t_{4}>t_{3}, D_{-}^{\alpha} x(t)>$ 0 , and $t \in\left[t_{4}, \infty\right)$. By Lemma 1 , we have

$$
\begin{aligned}
X(t) & -X\left(t_{4}\right)=\int_{t_{4}}^{t} X^{\prime}(s) d s \\
& =-\Gamma(1-\alpha) \int_{t_{4}}^{t} D_{-}^{\alpha} x(s) d s \\
& =-\Gamma(1-\alpha) \int_{t_{4}}^{t} \frac{r(s) D_{-}^{\alpha} x(s)}{r(s)} d s \\
& \leq-\Gamma(1-\alpha) r\left(t_{4}\right) D_{-}^{\alpha} x\left(t_{4}\right) \int_{t_{4}}^{t} \frac{1}{r(s)} d s .
\end{aligned}
$$

By (4), we obtain $\lim _{t \rightarrow \infty} X(t)=-\infty$, which contradicts $X(t)>0$ on $\left[t_{1}, \infty\right)$. So $\left[r(t) D^{\alpha} x(t)\right]^{\prime}<0$ on $\left[t_{2}, \infty\right)$. Thus $D^{\alpha} x(t)$ is eventually of one sign. Now we assume $D^{\alpha} x(t)>$ $0,{ }^{-} t \in\left[t_{5}, \infty\right)$ for some sufficiently $t_{5}>t_{4}$. Then by Lemma 1 , $X^{\prime}(t)<0$ for $t \in\left[t_{5}, \infty\right)$. Since $X(t)>0$, furthermore we have $\lim _{t \rightarrow \infty} X(t)=\beta \geq 0$. We claim $\beta=0$. Otherwise, assume $\beta>0$. Then $X(t) \geq \beta$ on $\left[t_{5}, \infty\right)$, and, for $t \in\left[t_{5}, \infty\right)$, by (7) we have

$$
\left(e^{A(t)} a(t)\left[r(t) D_{-}^{\alpha} x(t)\right]^{\prime}\right)^{\prime} \geq e^{A(t)} q(t) X(t) \geq \beta e^{A(t)} q(t) .
$$

Substituting $t$ with $s$ in (11), an integration for (11) with respect to $s$ from $t$ to $\infty$ yields

$$
\begin{aligned}
-e^{A(t)} & a(t)\left[r(t) D_{-}^{\alpha} x(t)\right]^{\prime} \\
& \geq-\lim _{t \rightarrow \infty} e^{A(t)} a(t)\left[r(t) D_{-}^{\alpha} x(t)\right]^{\prime}+\beta \int_{t}^{\infty} e^{A(s)} q(s) d s \\
& >\beta \int_{t_{5}}^{t} e^{A(s)} q(s) d s,
\end{aligned}
$$

which means

$$
\left(r(t) D_{-}^{\alpha} x(t)\right)^{\prime}<-\frac{\beta}{e^{A(t)} a(t)} \int_{t}^{\infty} e^{A(s)} q(s) d s .
$$

Substituting $t$ with $\tau$ in (13), an integration for (13) with respect to $\tau$ from $t$ to $\infty$ yields

$$
\begin{aligned}
-r(t) D_{-}^{\alpha} x(t)< & -\lim _{t \rightarrow \infty} r(t) D_{-}^{\alpha} x(t) \\
& -\beta \int_{t}^{\infty} \frac{1}{e^{A(\tau)} a(\tau)} \int_{\tau}^{\infty} e^{A(s)} q(s) d s d \tau \\
& <-\beta \int_{t}^{\infty} \frac{1}{e^{A(\tau)} a(\tau)} \int_{\tau}^{\infty} e^{A(s)} q(s) d s d \tau .
\end{aligned}
$$

That is,

$$
X^{\prime}(t)<-\frac{\Gamma(1-\alpha) \beta}{r(t)} \int_{t}^{\infty} \frac{1}{e^{A(\tau)} a(\tau)} \int_{\tau}^{\infty} e^{A(s)} q(s) d s d \tau .
$$


Substituting $t$ with $\xi$ in (15), an integration for (15) with respect to $\xi$ from $t_{5}$ to $t$ yields

$$
\begin{aligned}
& X(t)-X\left(t_{5}\right) \\
&<-\Gamma(1-\alpha) \beta \int_{t_{5}}^{t} \frac{1}{r(\xi)} \int_{\xi}^{\infty} \frac{1}{e^{A(\tau)} a(\tau)} \\
& \quad \times \int_{\tau}^{\infty} e^{A(s)} q(s) d s d \tau d \xi .
\end{aligned}
$$

By (5), one can see $\lim _{t \rightarrow \infty} X(t)=-\infty$, which is a contradiction. So the proof is complete.

Lemma 3. Assume that $x$ is an eventually positive solution of (2) such that

$$
\left[r(t) D_{-}^{\alpha} x(t)\right]^{\prime}<0, \quad D_{-}^{\alpha} x(t)<0 \quad \text { on }\left[t_{1}, \infty\right)
$$

where $t_{1} \geq t_{0}$ is sufficiently large. Then we have

$$
\begin{aligned}
& X^{\prime}(t) \geq-\frac{\Gamma(1-\alpha) \delta_{1}\left(t, t_{1}\right) e^{A(t)} a(t)\left(r(t) D_{-}^{\alpha} x(t)\right)^{\prime}}{r(t)}, \\
& X(t) \geq-\Gamma(1-\alpha) \delta_{2}\left(t, t_{1}\right) e^{A(t)} a(t)\left[r(t) D_{-}^{\alpha} x(t)\right]^{\prime}
\end{aligned}
$$

Proof. By Lemma 2 we have $e^{A(t)} a(t)\left[r(t) D^{\alpha} x(t)\right]^{\prime}$ is strictly increasing on $\left[t_{1}, \infty\right)$. So

$$
\begin{aligned}
r(t) D_{-}^{\alpha} x(t) & \leq r(t) D_{-}^{\alpha} x(t)-r\left(t_{1}\right) D_{-}^{\alpha} x(t) \\
& =\int_{t_{1}}^{t} \frac{e^{A(s)} a(s)\left[r(s) D_{-}^{\alpha} x(s)\right]^{\prime}}{e^{A(s)} a(s)} d s \\
& \leq e^{A(t)} a(t)\left[r(t) D_{-}^{\alpha} x(t)\right]^{\prime} \int_{t_{1}}^{t} \frac{1}{e^{A(s)} a(s)} d s \\
& =\delta_{1}\left(t, t_{1}\right) e^{A(t)} a(t)\left[r(t) D_{-}^{\alpha} x(t)\right]^{\prime} .
\end{aligned}
$$

Using Lemma 1 we obtain that

$$
X^{\prime}(t) \geq-\frac{\Gamma(1-\alpha) \delta_{1}\left(t, t_{1}\right) e^{A(t)} a(t)\left[r(t) D_{-}^{\alpha} x(t)\right]^{\prime}}{r(t)}
$$

Then

$$
\begin{aligned}
X(t) & \geq X(t)-X\left(t_{1}\right) \\
& \geq-\int_{t_{1}}^{t} \frac{\Gamma(1-\alpha) \delta_{1}\left(s, t_{1}\right) e^{A(s)} a(s)\left[r(s) D_{-}^{\alpha} x(s)\right]^{\prime}}{r(s)} d s \\
& \geq-\Gamma(1-\alpha) e^{A(t)} a(t)\left[r(t) D_{-}^{\alpha} x(t)\right]^{\prime} \int_{t_{1}}^{t} \frac{\delta_{1}\left(s, t_{1}\right)}{r(s)} d s \\
& =-\Gamma(1-\alpha) \delta_{2}\left(t, t_{1}\right) e^{A(t)} a(t)\left[r(t) D_{-}^{\alpha} x(t)\right]^{\prime} .
\end{aligned}
$$

Theorem 4. Assume (3)-(5) hold, and there exist two functions $\phi \in C^{1}\left(\left[t_{0}, \infty\right), \mathbb{R}_{+}\right)$and $\varphi \in C^{1}\left(\left[t_{0}, \infty\right),[0, \infty)\right)$ such that

$$
\begin{aligned}
\int_{T}^{\infty}\{\phi & (s) q(s) e^{A(s)}-\phi(s) \varphi^{\prime}(s) \\
& +\frac{\phi(s) \Gamma(1-\alpha) \delta_{1}(s, T) \varphi^{2}(s)}{r(s)} \\
& -\left[2 \varphi(s) \phi(s) \Gamma(1-\alpha) \delta_{1}(s, T)+r(s) \phi^{\prime}(s)\right]^{2} \\
& \left.\times\left(4 \Gamma(1-\alpha) \phi(s) \delta_{1}(s, T) r(s)\right)^{-1}\right\} d s=\infty,
\end{aligned}
$$

for all sufficiently large $T$. Then every solution of (2) is oscillatory or satisfies $\lim _{t \rightarrow \infty} X(t)=0$.

Proof. Assume (2) has a nonoscillatory solution $x$ on $\left[t_{0}, \infty\right)$. Without loss of generality, we may assume $x(t)>0$ on $\left[t_{1}, \infty\right)$, where $t_{1}$ is sufficiently large. By Lemma 2 we have $\left(r(t) D^{\alpha} x(t)\right)^{\prime}<0, t \in\left[t_{2}, \infty\right)$, where $t_{2}>t_{1}$ is sufficiently large, and either $D^{\alpha} x(t)<0$ on $\left[t_{2}, \infty\right)$ or $\lim _{t \rightarrow \infty} X(t)=0$. Define the generalized Riccati function:

$$
\omega(t)=\phi(t)\left\{-\frac{e^{A(t)} a(t)\left[r(t) D_{-}^{\alpha} x(t)\right]^{\prime}}{X(t)}+\varphi(t)\right\} .
$$

Then for $t \in\left[t_{2}, \infty\right)$, we have

$$
\begin{aligned}
\omega^{\prime}(t)= & -\phi^{\prime}(t) \frac{e^{A(t)} a(t)\left[r(t) D_{-}^{\alpha} x(t)\right]^{\prime}}{X(t)} \\
& +\phi(t)\left\{-\frac{e^{A(t)} a(t)\left[r(t) D_{-}^{\alpha} x(t)\right]^{\prime}}{X(t)}\right\} \\
& +\phi^{\prime}(t) \varphi(t)+\phi(t) \varphi^{\prime}(t) \\
= & -\phi(t)\left\{\left(X(t)\left(e^{A(t)} a(t)\left[r(t) D_{-}^{\alpha} x(t)\right]^{\prime}\right)^{\prime}\right.\right. \\
& \left.-X^{\prime}(t) e^{A(t)} a(t)\left[r(t) D^{\alpha} x(t)\right]^{\prime}\right) \\
& +\frac{\phi^{\prime}(t)}{\phi(t)} \omega(t)+\phi(t) \varphi^{\prime}(t) \\
& +\frac{\phi^{\prime}(t)}{\phi(t)} \omega(t)+\phi(t) \varphi^{\prime}(t) \\
= & \quad \phi(t)\left\{\left(X ( t ) \left\{e^{A(t)}\left(a(t)\left[r(t) D_{-}^{\alpha} x(t)\right]^{\prime}\right)^{\prime}\right.\right.\right. \\
& \left.\quad-X^{\prime}(t) e^{A(t)} a(t)\left[r(t) D^{\alpha} x(t)\right]^{\prime}\right) \\
&
\end{aligned}
$$




$$
\begin{aligned}
= & -\phi(t) q(t) e^{A(t)} \\
& +\frac{\phi(t) X^{\prime}(t) a(t) e^{A(t)}\left[r(t) D_{-}^{\alpha} x(t)\right]^{\prime}}{X^{2}(t)} \\
& +\frac{\phi^{\prime}(t)}{\phi(t)} \omega(t)+\phi(t) \varphi^{\prime}(t) .
\end{aligned}
$$

By Lemma 3 and the definition of $f$ we get that

$$
\begin{aligned}
& \omega^{\prime}(t) \leq-\phi(t) q(t) e^{A(t)} \\
& -\left(\phi(t) \Gamma(1-\alpha) \delta_{1}\left(t, t_{2}\right) e^{A(t)} a(t)\right. \\
& \left.\times\left(r(t) D_{-}^{\alpha} x(t)\right)^{\prime} a(t) e^{A(t)}\left(r(t) D_{-}^{\alpha} x(t)\right)^{\prime}\right) \\
& \times\left(r(t) X^{2}(t)\right)^{-1}+\frac{\phi^{\prime}(t)}{\phi(t)} \omega(t)+\phi(t) \varphi^{\prime}(t) \\
& =-\phi(t) q(t) e^{A(t)}-\frac{\phi(t) \Gamma(1-\alpha) \delta_{1}\left(t, t_{2}\right)}{r(t)} \\
& \times\left[\frac{\omega(t)}{\phi(t)}-\varphi(t)\right]^{2}+\frac{\phi^{\prime}(t)}{\phi(t)} \omega(t)+\phi(t) \varphi^{\prime}(t) \\
& =-\phi(t) q(t) e^{A(t)}+\phi(t) \varphi^{\prime}(t) \\
& -\frac{\phi(t) \Gamma(1-\alpha) \delta_{1}\left(t, t_{2}\right) \varphi^{2}(t)}{r(t)} \\
& -\frac{\phi(t) \Gamma(1-\alpha) \delta_{1}\left(t, t_{2}\right)}{r(t)} \frac{\omega^{2}(t)}{\phi^{2}(t)} \\
& +\frac{2 \varphi(t) \phi(t) \Gamma(1-\alpha) \delta_{1}\left(t, t_{2}\right)+r(t) \phi^{\prime}(t)}{r(t) \phi(t)} \omega(t) \\
& \leq-\phi(t) q(t) e^{A(t)}+\phi(t) \varphi^{\prime}(t) \\
& -\frac{\phi(t) \Gamma(1-\alpha) \delta_{1}\left(t, t_{2}\right) \varphi^{2}(t)}{r(t)} \\
& +\frac{\left[2 \varphi(t) \phi(t) \Gamma(1-\alpha) \delta_{1}\left(t, t_{2}\right)+r(t) \phi^{\prime}(t)\right]^{2}}{4 \Gamma(1-\alpha) \phi(t) \delta_{1}\left(t, t_{2}\right) r(t)} .
\end{aligned}
$$

Substituting $t$ with $s$ in (26), an integration for (26) with respect to $s$ from $t_{2}$ to $t$ yields

$$
\begin{aligned}
\int_{t_{2}}^{t}\{\phi(s) & q(s) e^{A(s)}-\phi(s) \varphi^{\prime}(s) \\
+ & \frac{\phi(s) \Gamma(1-\alpha) \delta_{1}\left(s, t_{2}\right) \varphi^{2}(s)}{r(s)} \\
& -\left[2 \varphi(s) \phi(s) \Gamma(1-\alpha) \delta_{1}\left(s, t_{2}\right)+r(s) \phi^{\prime}(s)\right]^{2}
\end{aligned}
$$

$$
\begin{aligned}
& \left.\times\left(4 \Gamma(1-\alpha) \phi(s) \delta_{1}\left(s, t_{2}\right) r(s)\right)^{-1}\right\} d s \\
\leq & \omega\left(t_{2}\right)-\omega(t) \leq \omega\left(t_{2}\right)<\infty,
\end{aligned}
$$

which contradicts (23). So the proof is complete.

Theorem 5. Define $\mathbb{D}=\left\{(t, s) \mid t \geq s \geq t_{0}\right\}$. Assume (3)-(5) hold, and there exists a function $H \in C^{1}(\mathbb{D}, \mathbb{R})$ such that

$$
H(t, t)=0, \quad \text { for } t \geq t_{0}, \quad H(t, s)>0, \quad \text { for } t>s \geq t_{0} \text {, }
$$

and $H$ has a nonpositive continuous partial derivative $H_{s}^{\prime}(t, s)$, and

$$
\begin{aligned}
& \lim _{t \rightarrow \infty} \sup \frac{1}{H\left(t, t_{0}\right)} \\
& \times\left\{\int _ { t _ { 0 } } ^ { t } H ( t , s ) \left\{\phi(s) q(s) e^{A(s)}-\phi(s) \varphi^{\prime}(s)\right.\right. \\
&+\frac{\phi(s) \Gamma(1-\alpha) \delta_{1}(s, T) \varphi^{2}(s)}{r(s)} \\
&-[2 \varphi(s) \phi(s) \Gamma(1-\alpha) \\
& \times\left(4 \Gamma(1-\alpha) \phi(s) \delta_{1}(s, T)+r(s) \phi^{\prime}(s)\right]^{2} \\
&\left.\left.\times(s, T) r(s))^{-1}\right\} d s\right\}=\infty,
\end{aligned}
$$

for all sufficiently large $T$, where $\phi, \varphi$ are defined as in Theorem 4. Then every solution of (2) is oscillatory or satisfies $\lim _{t \rightarrow \infty} X(t)=0$.

Proof. Assume (2) has a nonoscillatory solution $x$ on $\left[t_{0}, \infty\right)$. Without loss of generality, we may assume $x(t)>0$ on $\left[t_{1}, \infty\right)$, where $t_{1}$ is sufficiently large. By Lemma 2 we have $D^{\alpha} x(t)<0$ on $\left[t_{2}, \infty\right)$ for some sufficiently large $t_{2}>t_{1}$. Let $\bar{\omega}(t)$ be defined as in Theorem 4. By (26) we have

$$
\begin{gathered}
\phi(t) q(t) e^{A(t)}+\phi(t) \varphi^{\prime}(t)-\frac{\phi(t) \Gamma(1-\alpha) \delta_{1}\left(t, t_{2}\right) \varphi^{2}(t)}{r(t)} \\
+\frac{\left[2 \varphi(t) \phi(t) \Gamma(1-\alpha) \delta_{1}\left(t, t_{2}\right)+r(t) \phi^{\prime}(t)\right]^{2}}{4 \Gamma(1-\alpha) \phi(t) \delta_{1}\left(t, t_{2}\right) r(t)} \leq-\omega^{\prime}(t) .
\end{gathered}
$$

Substituting $t$ with $s$ in (30), multiplying both sides by $H(t, s)$, and then integrating it with respect to $s$ from $t_{2}$ to $t$ yield

$$
\begin{aligned}
& \int_{t_{2}}^{t} H(t, s) \\
& \quad \times\left\{\begin{array}{l}
\phi(s) q(s) e^{A(s)}-\phi(s) \varphi^{\prime}(s) \\
\quad+\frac{\phi(s) \Gamma(1-\alpha) \delta_{1}\left(s, t_{2}\right) \varphi^{2}(s)}{r(s)}
\end{array}\right.
\end{aligned}
$$




$$
\begin{aligned}
& \left.-\frac{\left[2 \varphi(s) \phi(s) \Gamma(1-\alpha) \delta_{1}\left(s, t_{2}\right)+r(s) \phi^{\prime}(s)\right]^{2}}{4 \Gamma(1-\alpha) \phi(s) \delta_{1}\left(s, t_{2}\right) r(s)}\right\} d s \\
\leq- & \int_{t_{2}}^{t} H(t, s) \omega^{\prime}(s) d s \\
= & H\left(t, t_{2}\right) \omega\left(t_{2}\right)+\int_{t_{2}}^{t} H_{s}^{\prime}(t, s) \omega(s) \Delta s \\
\leq & H\left(t, t_{2}\right) \omega\left(t_{2}\right) \leq H\left(t, t_{0}\right) \omega\left(t_{2}\right) .
\end{aligned}
$$

Then

$$
\begin{aligned}
& \int_{t_{0}}^{t} H(t, s) \\
& \times\left\{\phi(s) q(s) e^{A(s)}-\phi(s) \varphi^{\prime}(s)\right. \\
& +\frac{\phi(s) \Gamma(1-\alpha) \delta_{1}\left(s, t_{2}\right) \varphi^{2}(s)}{r(s)} \\
& \text { - }[2 \varphi(s) \phi(s) \Gamma(1-\alpha) \\
& \left.\times \delta_{1}\left(s, t_{2}\right)+r(s) \phi^{\prime}(s)\right]^{2} \\
& \left.\times\left(4 \Gamma(1-\alpha) \phi(s) \delta_{1}\left(s, t_{2}\right) r(s)\right)^{-1}\right\} d s \\
& =\int_{t_{0}}^{t_{2}} H(t, s) \\
& \times\left\{\phi(s) q(s) e^{A(s)}-\phi(s) \varphi^{\prime}(s)\right. \\
& +\frac{\phi(s) \Gamma(1-\alpha) \delta_{1}\left(s, t_{2}\right) \varphi^{2}(s)}{r(s)} \\
& \left.-\frac{\left[2 \varphi(s) \phi(s) \Gamma(1-\alpha) \delta_{1}\left(s, t_{2}\right)+r(s) \phi^{\prime}(s)\right]^{2}}{4 \Gamma(1-\alpha) \phi(s) \delta_{1}\left(s, t_{2}\right) r(s)}\right\} d s \\
& +\int_{t_{2}}^{t} H(t, s) \\
& \times\left\{\phi(s) q(s) e^{A(s)}-\phi(s) \varphi^{\prime}(s)\right. \\
& +\frac{\phi(s) \Gamma(1-\alpha) \delta_{1}\left(s, t_{2}\right) \varphi^{2}(s)}{r(s)} \\
& \left.-\frac{\left[2 \varphi(s) \phi(s) \Gamma(1-\alpha) \delta_{1}\left(s, t_{2}\right)+r(s) \phi^{\prime}(s)\right]^{2}}{4 \Gamma(1-\alpha) \phi(s) \delta_{1}\left(s, t_{2}\right) r(s)}\right\}
\end{aligned}
$$$$
\times d s
$$

$$
\begin{aligned}
\leq H\left(t, t_{0}\right) \omega\left(t_{2}\right)+H\left(t, t_{0}\right) \\
\times \int_{t_{0}}^{t_{2}} \mid \phi(s) q(s) e^{A(s)}-\phi(s) \varphi^{\prime}(s) \\
+\left(\phi(s) \Gamma(1-\alpha) \delta_{1}\left(s, t_{2}\right) \varphi^{2}(s)\right) \\
\times(r(s))^{-1} \\
-\left[2 \varphi(s) \phi(s) \Gamma(1-\alpha) \delta_{1}\right. \\
\left.\times\left(s, t_{2}\right)+r(s) \phi^{\prime}(s)\right]^{2} \\
\times\left(4 \Gamma(1-\alpha) \phi(s) \delta_{1}\left(s, t_{2}\right) r(s)\right)^{-1} \mid d s .
\end{aligned}
$$

So

$$
\begin{gathered}
\lim _{t \rightarrow \infty} \sup \frac{1}{H\left(t, t_{0}\right)} \\
\times\left\{\int _ { t _ { 0 } } ^ { t } H ( t , s ) \left\{\phi(s) q(s) e^{A(s)}-\phi(s) \varphi^{\prime}(s)\right.\right. \\
+\left(\phi(s) \Gamma(1-\alpha) \delta_{1}\left(s, t_{2}\right) \varphi^{2}(s)\right) \\
\quad \times(r(s))^{-1}-[2 \varphi(s) \phi(s) \Gamma(1-\alpha) \\
\left.\times \delta_{1}\left(s, t_{2}\right)+r(s) \phi^{\prime}(s)\right]^{2} \\
\left.\left.\quad \times\left(4 \Gamma(1-\alpha) \phi(s) \delta_{1}\left(s, t_{2}\right) r(s)\right)^{-1}\right\} d s\right\} \\
\leq \omega\left(t_{2}\right) \quad \\
+\int_{t_{0}}^{t_{2}} \mid \phi(s) q(s) e^{A(s)}-\phi(s) \varphi^{\prime}(s) \\
+\frac{\phi(s) \Gamma(1-\alpha) \delta_{1}\left(s, t_{2}\right) \varphi^{2}(s)}{r(s)} \\
-\left[2 \varphi(s) \phi(s) \Gamma(1-\alpha) \delta_{1}\left(s, t_{2}\right)+r(s) \phi^{\prime}(s)\right]^{2} \\
\times\left(4 \Gamma(1-\alpha) \phi(s) \delta_{1}\left(s, t_{2}\right) r(s)\right)^{-1} \mid d s<\infty
\end{gathered}
$$

which contradicts (29). So the proof is complete.

In Theorems 5 , if we take $H(t, s)$ for some special functions such as $(t-s)^{m}$ or $\ln (t / s)$, then we can obtain some corollaries as follows.

Corollary 6. Assume (3)-(5) hold, and

$$
\begin{aligned}
& \lim _{t \rightarrow \infty} \sup \frac{1}{\left(t-t_{0}\right)^{m}} \\
& \times\left\{\int _ { t _ { 0 } } ^ { t } ( t - s ) ^ { m } \left\{\phi(s) q(s) e^{A(s)}-\phi(s) \varphi^{\prime}(s)\right.\right. \\
&+\left(\phi(s) \Gamma(1-\alpha) \delta_{1}(s, T) \varphi^{2}(s)\right)
\end{aligned}
$$




$$
\begin{aligned}
& \times(r(s))^{-1} \\
& -\left[2 \varphi(s) \phi(s) \Gamma(1-\alpha) \delta_{1}(s, T)\right. \\
& \left.\quad+r(s) \phi^{\prime}(s)\right]^{2} \\
& \times\left(4 \Gamma(1-\alpha) \phi(s) \delta_{1}\right. \\
& \left.\left.\quad \times(s, T) r(s))^{-1}\right\} d s\right\}=\infty,
\end{aligned}
$$

for all sufficiently large $T$. Then every solution of (2) is oscillatory or satisfies $\lim _{t \rightarrow \infty} X(t)=0$.

Corollary 7. Assume (3)-(5) hold, and

$$
\begin{aligned}
& \lim _{t \rightarrow \infty} \sup \frac{1}{\left(\ln t-\ln t_{0}\right)} \\
& \times\left\{\int _ { t _ { 0 } } ^ { t } ( \operatorname { l n } t - \operatorname { l n } s ) \left\{\phi(s) q(s) e^{A(s)}-\phi(s) \varphi^{\prime}(s)\right.\right. \\
&+\frac{\phi(s) \Gamma(1-\alpha) \delta_{1}(s, T) \varphi^{2}(s)}{r(s)} \\
&-\left[2 \varphi(s) \phi(s) \Gamma(1-\alpha) \delta_{1}(s, T)\right. \\
& \quad \times(4 \Gamma(1-\alpha) \phi(s) \\
&\left.\left.\left.\quad \times \delta_{1}(s, T) r(s)\right)^{-1}\right\} d s\right\}=\infty
\end{aligned}
$$

for all sufficiently large $T$. Then every solution of (2) is oscillatory or satisfies $\lim _{t \rightarrow \infty} X(t)=0$.

\section{Applications}

In this section, we will present some applications for the above established results.

Example 8. Consider

$$
\begin{aligned}
& \left(t\left(D_{-}^{\alpha} x(t)\right)^{\prime}\right)^{\prime}+t^{-2}\left(D_{-}^{\alpha} x(t)\right)^{\prime} \\
& \quad-M t^{-2} \int_{t}^{\infty}(\xi-t)^{-\alpha} x(\xi) d \xi=0, \quad t \in[2, \infty),
\end{aligned}
$$

where $M>0$ is a quotient of two odd positive integers.

We have in (2) $a(t)=t, p(t)=t^{-2}, q(t)=M t^{-2}, r(t)=$ 1 , and $t_{0}=2$. Then

$$
1 \leq e^{A(t)}=e^{\int_{2}^{t}(p(s) / a(s)) d s}=e^{\int_{2}^{t} s^{-3} d s}=e^{-(1 / 2)\left[t^{-2}-2^{-2}\right]} \leq e^{1 / 8} .
$$

Moreover, we have

$$
\begin{aligned}
\int_{t_{0}}^{\infty} \frac{1}{e^{A(s)} a(s)} d s= & \int_{2}^{\infty} \frac{1}{e^{A(s)} a(s)} d s \geq e^{-1 / 8} \int_{2}^{\infty} \frac{1}{s} d s=\infty, \\
& \int_{t_{0}}^{\infty} \frac{1}{r(s)} d s=\infty
\end{aligned}
$$

Furthermore,

$$
\begin{gathered}
\int_{t_{0}}^{\infty} \frac{1}{r(\xi)} \int_{\xi}^{\infty} \frac{1}{e^{A(\tau)} a(\tau)} \int_{\tau}^{\infty} e^{A(s)} q(s) d s d \tau d \xi \\
\quad=M \int_{2}^{\infty} \int_{\xi}^{\infty} \frac{1}{e^{A(\tau)} \tau} \int_{\tau}^{\infty} \frac{e^{A(s)}}{s^{2}} d s d \tau d \xi \\
\geq M e^{-1 / 8} \int_{2}^{\infty} \int_{\xi}^{\infty} \frac{1}{\tau} \int_{\tau}^{\infty} \frac{1}{s^{2}} d s d \tau d \xi \\
\quad=M e^{-1 / 8} \int_{2}^{\infty} \int_{\xi}^{\infty} \frac{1}{\tau^{2}} d \tau d \xi \\
=M e^{-1 / 8} \int_{2}^{\infty} \frac{1}{\xi} d \xi=\infty .
\end{gathered}
$$

On the other hand, for a sufficiently large $T$, we have

$$
\begin{aligned}
\delta_{1}(t, T) & =\int_{T}^{t} \frac{1}{e^{A(s)} a(s)} d s \\
& =\int_{T}^{t} \frac{1}{e^{A(s)} s} d s \geq e^{-1 / 8} \int_{T}^{t} \frac{1}{s} d s \longrightarrow \infty .
\end{aligned}
$$

So we can take $T^{*}>T$ such that $\delta_{1}(t, T)>1$ for $t \in\left[T^{*}, \infty\right)$. Taking $\phi(t)=t, \varphi(t)=0$ in (23), we get that

$$
\begin{gathered}
\int_{T}^{\infty}\left\{\phi(s) q(s) e^{A(s)}-\frac{\left[r(s) \phi^{\prime}(s)\right]^{2}}{4\left[\Gamma(1-\alpha) \phi(s) \delta_{1}(s, T)\right] r(s)}\right\} d s \\
\geq \int_{T}^{\infty}\left[M-\frac{1}{4 \Gamma(1-\alpha) \delta_{1}(s, T)}\right] \frac{1}{s} d s \\
=\int_{T}^{T^{*}}\left[M-\frac{1}{4 \Gamma(1-\alpha) \delta_{1}(s, T)}\right] \frac{1}{s} d s \\
\geq \int_{T}^{T^{*}}\left[M-\frac{1}{4 \Gamma(1-\alpha) \delta_{1}(s, T)}\right] \frac{1}{s} d s \\
\quad+\int_{T^{*}}^{\infty}\left[M-\frac{1}{4 \Gamma(1-\alpha) \delta_{1}(s, T)}\right] \frac{1}{s} d s \\
M\left[M-\frac{1}{4 \Gamma(1-\alpha)} d s=\infty,\right.
\end{gathered}
$$

provided that $M>1 /(4 \Gamma(1-\alpha))$. So (3)-(5) and (23) all hold, and by Theorem 4 we deduce that every solution of (36) is oscillatory or satisfies $\lim _{t \rightarrow \infty} X(t)=0$ under condition $M>$ $1 / 4 \Gamma(1-\alpha)$. 
Example 9. Consider

$$
\begin{aligned}
& \left(\sqrt{t}\left(D_{-}^{\alpha} x(t)\right)^{\prime}\right)^{\prime}+e^{-t}\left(D_{-}^{\alpha} x(t)\right)^{\prime} \\
& -\left(M t^{-5 / 2}+\ln t\right) \int_{t}^{\infty}(\xi-t)^{-\alpha} x(\xi) d \xi=0, \quad t \in[2, \infty),
\end{aligned}
$$

where $\alpha \in(0,1)$, and $M>0$ is a constant.

We have in (2) $a(t)=\sqrt{t}, p(t)=e^{-t}, q(t)=M t^{-5 / 2}+$ $\ln t, r(t)=1$, and $t_{0}=2$. Then

$$
\begin{aligned}
1 & \leq e^{A(t)}=e^{\int_{2}^{t}(p(s) / a(s)) d s}=e^{\int_{2}^{t}\left(1 / e^{s} \sqrt{s}\right) d s} \\
& \leq e^{\int_{2}^{t} s^{-(3 / 2) d s}}=e^{-2\left[t^{-1 / 2}-2^{-1 / 2}\right]} \leq e^{\sqrt{2}} .
\end{aligned}
$$

So we have

$$
\begin{aligned}
\int_{t_{0}}^{\infty} \frac{1}{e^{A(s)} a(s)} d s= & \int_{2}^{\infty} \frac{1}{e^{A(s)} \sqrt{s}} d s \geq e^{-\sqrt{2}} \int_{2}^{\infty} \frac{1}{\sqrt{s}} d s=\infty, \\
& \int_{t_{0}}^{\infty} \frac{1}{r(s)} d s=\infty
\end{aligned}
$$

Furthermore,

$$
\begin{aligned}
\int_{t_{0}}^{\infty} \frac{1}{r(\xi)} \int_{\xi}^{\infty} \frac{1}{e^{A(\tau)} a(\tau)} \int_{\tau}^{\infty} e^{A(s)} q(s) d s d \tau d \xi \\
\quad=\int_{2}^{\infty} \int_{\xi}^{\infty} \frac{1}{e^{A(\tau)} \sqrt{\tau}} \int_{\tau}^{\infty} e^{A(s)}\left(M s^{-5 / 2}+\ln s\right) d s d \tau d \xi \\
\geq M e^{-\sqrt{2}} \int_{2}^{\infty} \int_{\xi}^{\infty} \frac{1}{\sqrt{\tau}} \int_{\tau}^{\infty} s^{-5 / 2} d s d \tau d \xi \\
=\frac{2 M e^{-\sqrt{2}}}{3} \int_{2}^{\infty}\left[\int_{\xi}^{\infty} \frac{1}{\tau^{2}} d \tau\right] d \xi \\
=\frac{2 M e^{-\sqrt{2}}}{3} \int_{2}^{\infty} \frac{1}{\xi} d \xi=\infty
\end{aligned}
$$

On the other hand, Taking $\phi(t)=t^{2}, \varphi(t)=0$, and $H(t, s)=$ $t-s$ in (29), we get that

$$
\begin{aligned}
\lim _{t \rightarrow \infty} \sup \frac{1}{t-t_{0}}\left\{\int_{t_{0}}^{t}(t-s)\right. & \\
\quad & \left\{\phi(s) q(s) e^{A(s)}\right. \\
& \left.\left.\quad-\frac{\left[r(s) \phi^{\prime}(s)\right]^{2}}{4 \Gamma(1-\alpha) \phi(s) \delta_{1}(s, T) r(s)}\right\} d s\right\}
\end{aligned}
$$

$$
\begin{aligned}
& \geq \lim _{t \rightarrow \infty} \sup \frac{1}{t-2} \\
& \quad \times\left\{\int _ { 2 } ^ { t } ( t - s ) \left\{\left(M s^{-5 / 2}+\ln s\right) s^{2}\right.\right. \\
& \left.\left.\quad-\frac{1}{\Gamma(1-\alpha) \delta_{1}(s, T)}\right\} d s\right\} \\
& \geq \lim _{t \rightarrow \infty} \sup \frac{1}{t-2}\left\{\int_{2}^{t}(t-s) M s^{-1 / 2} d s\right\}=\infty .
\end{aligned}
$$

So (3)-(5) and (29) all hold, and by Corollary 6 with $m=1$ we deduce that every solution of (42) is oscillatory or satisfies $\lim _{t \rightarrow \infty} X(t)=0$.

\section{Acknowledgments}

This work is partially supported by Planning Fund project of the Ministry of Education of China (10YJA630019). The authors would thank the reviewers very much for their valuable suggestions on this paper.

\section{References}

[1] A. Saadatmandi and M. Dehghan, "A new operational matrix for solving fractional-order differential equations," Computers \& Mathematics with Applications, vol. 59, no. 3, pp. 1326-1336, 2010.

[2] Y. Zhou, F. Jiao, and J. Li, "Existence and uniqueness for p-type fractional neutral differential equations," Nonlinear Analysis. Theory, Methods \& Applications, vol. 71, no. 7-8, pp. 2724-2733, 2009.

[3] L. Galeone and R. Garrappa, "Explicit methods for fractional differential equations and their stability properties," Journal of Computational and Applied Mathematics, vol. 228, no. 2, pp. 548-560, 2009.

[4] J. C. Trigeassou, N. Maamri, J. Sabatier, and A. Oustaloup, "A Lyapunov approach to the stability of fractional differential equations," Signal Process, vol. 91, pp. 437-445, 2011.

[5] W. Deng, "Smoothness and stability of the solutions for nonlinear fractional differential equations," Nonlinear Analysis. Theory, Methods \& Applications, vol. 72, no. 3-4, pp. 1768-1777, 2010.

[6] F. Ghoreishi and S. Yazdani, "An extension of the spectral Tau method for numerical solution of multi-order fractional differential equations with convergence analysis," Computers \& Mathematics with Applications, vol. 61, no. 1, pp. 30-43, 2011.

[7] J. T. Edwards, N. J. Ford, and A. C. Simpson, “The numerical solution of linear multi-term fractional differential equations; systems of equations," Journal of Computational and Applied Mathematics, vol. 148, no. 2, pp. 401-418, 2002.

[8] M. Muslim, "Existence and approximation of solutions to fractional differential equations," Mathematical and Computer Modelling, vol. 49, no. 5-6, pp. 1164-1172, 2009.

[9] D.-X. Chen, "Oscillation criteria of fractional differential equations," Advances in Difference Equations, vol. 2012, article 33, 2012.

[10] I. Podlubny, Fractional Differential Equations, vol. 198, Academic Press, San Diego, Calif, USA, 1999. 
[11] A. A. Kilbas, H. M. Srivastava, and J. J. Trujillo, Theory and Applications of Fractional Differential Equations, vol. 204, Elsevier Science B.V., Amsterdam, The Netherlands, 2006.

[12] S. Das, Functional Fractional Calculus, Springer, Berlin, Germany, 2nd edition, 2011. 


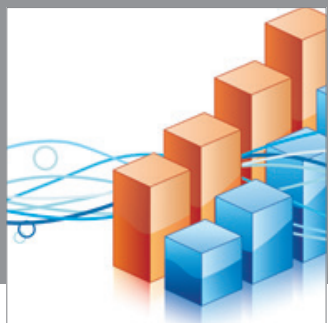

Advances in

Operations Research

mansans

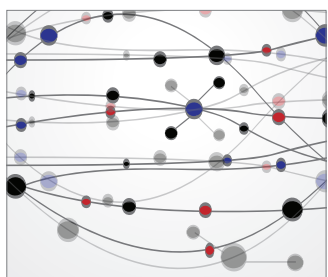

The Scientific World Journal
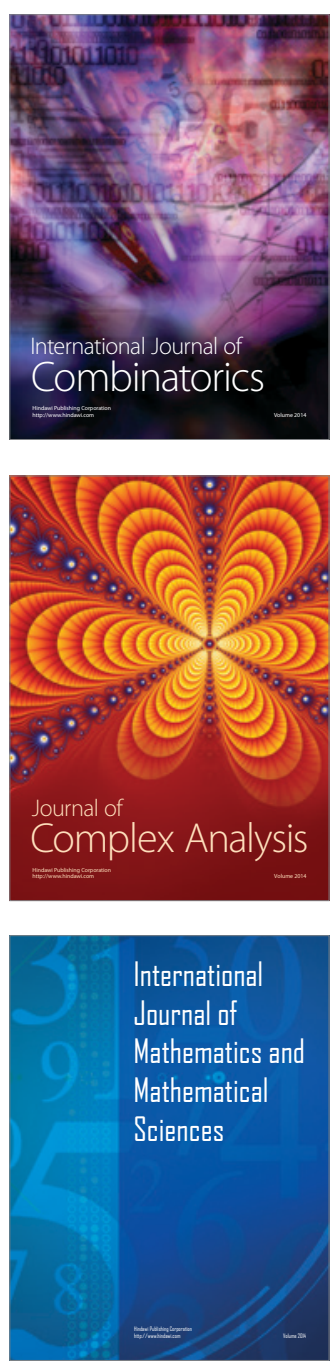
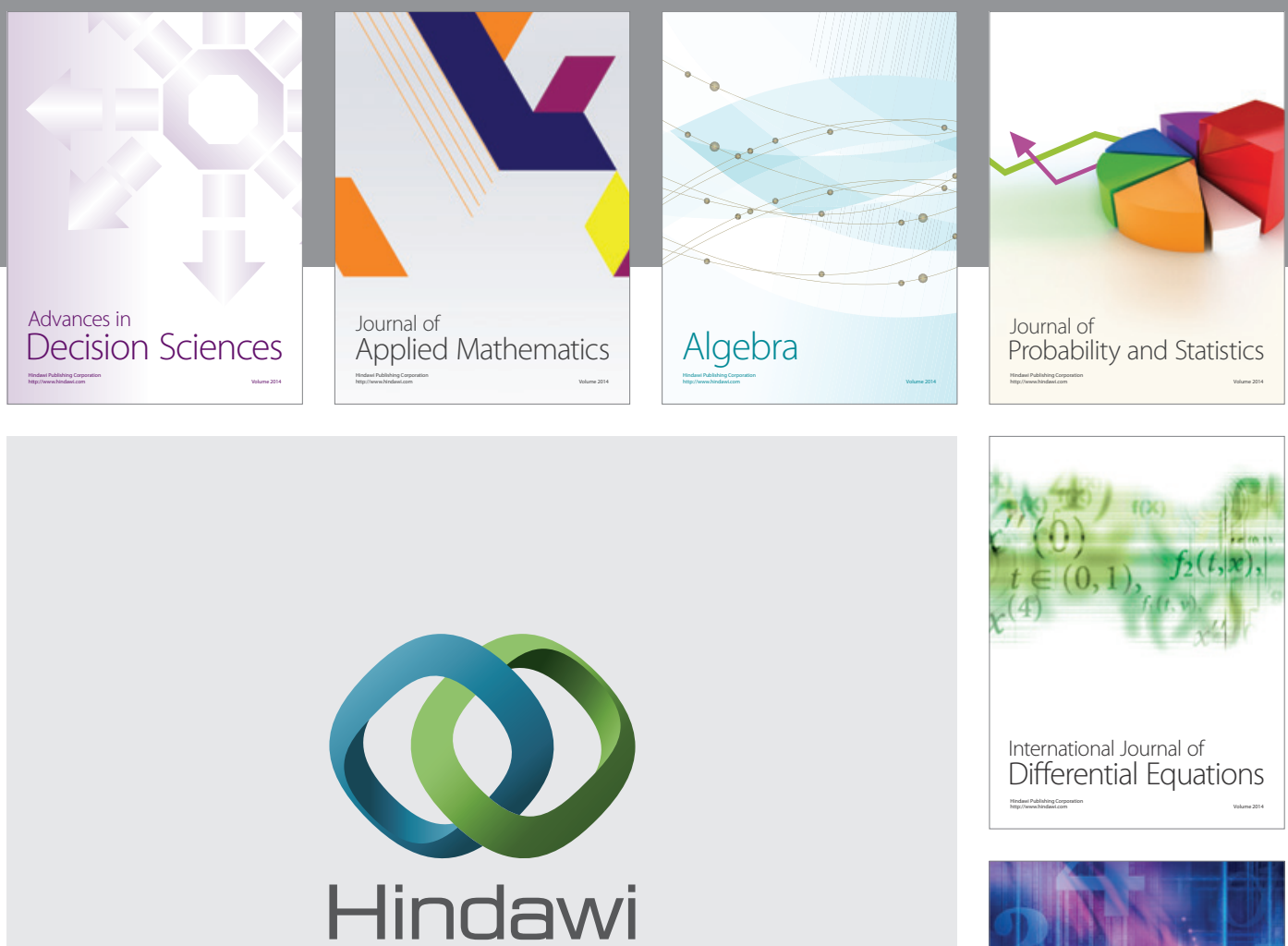

Submit your manuscripts at http://www.hindawi.com
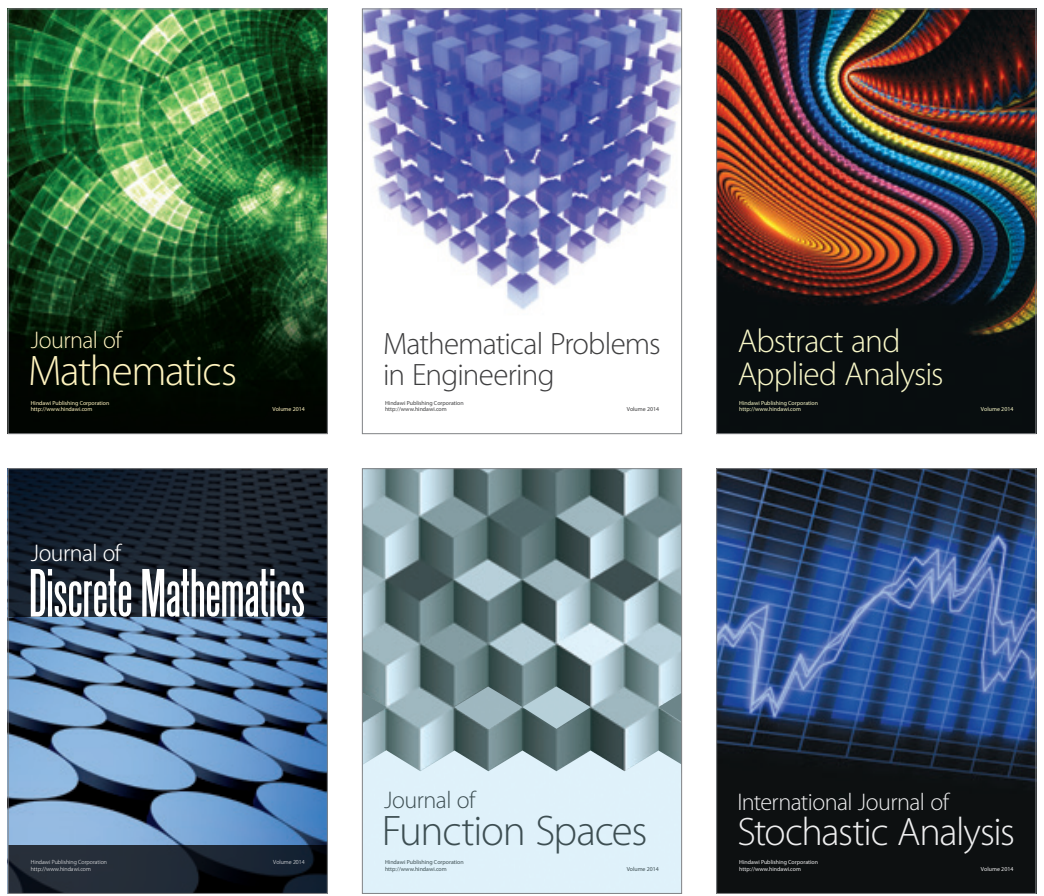

Journal of

Function Spaces

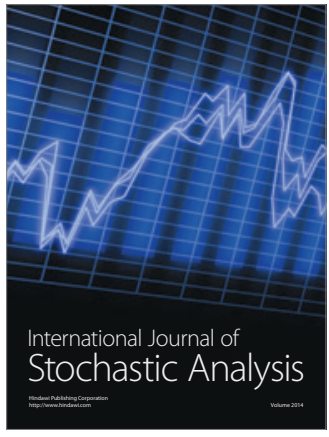

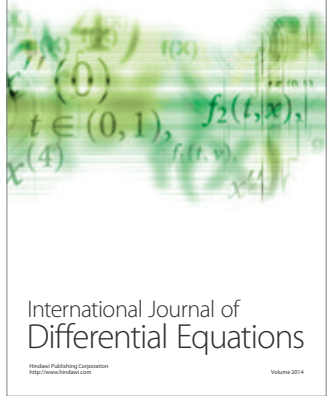
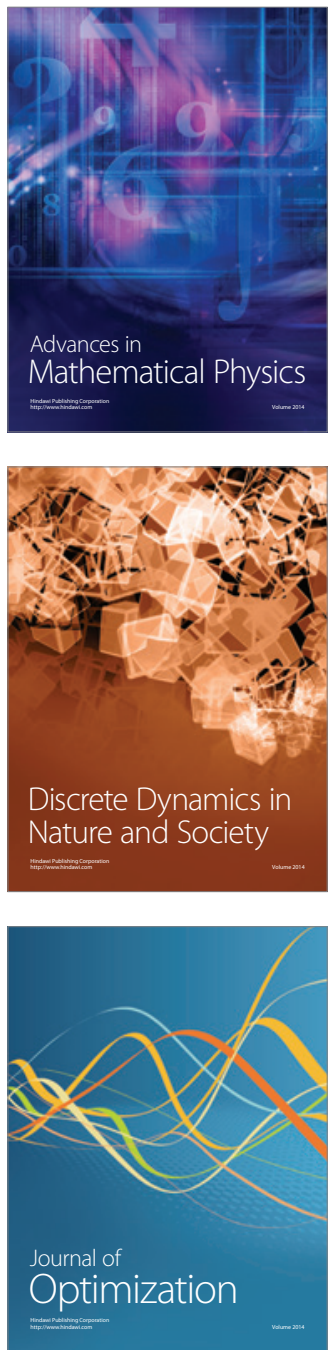